\title{
A model to improve the appropriateness in the management of acute poly-pathological patients: the Acute Complex Care Model
}

\author{
Francesco Orlandini, ${ }^{1}$ Filomena Pietrantonio, ${ }^{2}$ Luca Moriconi, ${ }^{3}$ Micaela La Regina, ${ }^{1}$ Antonino Mazzone, ${ }^{4}$ \\ Mauro Campanini ${ }^{5}$
}

${ }^{1}$ Department of Internal Medicine, East Ligurian Hospital, ASL5 Liguria, La Spezia; ${ }^{2}$ Department of Internal Medicine, Manerbio Hospital (BS), ASST-Garda; ${ }^{3}$ Department of Internal Medicine, S. Giovanni-Addolorata Hospital, Roma; ${ }^{4}$ Medical Area Department, Hospital of Legnano (MI); ${ }^{5}$ Medical Department, University Hospital Maggiore della Carità,

Novara, Italy

\begin{abstract}
Improved care for acute diseases has increased mean age and the proportion of people affected by multiple chronic diseases, while mutated socioeconomic conditions augmented the number of elderly and socially frail subjects. Multi-morbid patients who require a global approach not-fragmented-care interventions and a close connection between health and social services are progressively growing. Acute Complex Care Model (ACCM) is a model of integrated management of the frail patients when they need acute hospital care. The name of our model - ACCM - just recalls the chronic care model (CCM), designed for the de-hospitalization of chronic patients by preventing acute exacerbations, representing the hospital counterpart of the CCM. The target population is made up of acutely ill complex and poly-pathological patients (AICPPs), admitted to hospital and requiring high technological resources and continuous monitoring; the mission is to improve the management of medical admissions through pre-defined intra-hospital tracks and a global, multidisciplinary, patient-centered approach. Nowadays, multiple uncoordinated specialists care for AICPPs who move from one ward to another, with dangerous loss of information and continuity. ACCM tries to overcome these problems by putting the internal medicine specialist as the only referent for that patient and the coordinator of the care team. According to a model of future hospital organized with different intensity settings, the Internal Medicine Wards, caring for AICPPS, are organized in High Dependency Areas and Ordinary Areas. For both we present organizational and personnel standards that are lacking in daily practice. The epidemiological transition leading to a progressive increase in AICPPs requiring frequent hospitalization enhances the role of hospital internal medicine specialist in the coordination and delivery of care. The ACCM represents a practical response to this epochal change of roles. Medical and nursing staff standards and competencies have to be reviewed to ensure adequate care for these patients.
\end{abstract}

Correspondence: Filomena Pietrantonio, Department of Internal Medicine, Manerbio Hospital (BS), ASST-Garda, via Lungomella Valsecchi 2, 25025 Manerbio (BS), Italy.

E-mail: filomena.pietrantonio@gmail.com

Key words: Acute care; polypathological patients; internal medicine; medical ward; comorbidities.

Contributions: the authors contributed equally.

Conflict of interest: the authors declare no potential conflict of interest.

Received for publication: 24 January 2016.

Revision received: 29 February 2016.

Accepted for publication: 23 March 2016.

This work is licensed under a Creative Commons Attribution NonCommercial 4.0 License (CC BY-NC 4.0).

CCopyright F. Orlandini et al., 2017

Licensee PAGEPress, Italy

Italian Journal of Medicine 2017; 11:1-7

doi:10.4081/itjm.2016.697

\section{Introduction}

Improved care for acute diseases has increased mean age and the proportion of people affected by multiple chronic diseases, while mutated socioeconomic conditions augmented the number of elderly and socially frail subjects.

According to the World Health Organization (WHO), cardiovascular diseases, cancer, chronic obstructive pulmonary disease, and diabetes account for nearly $86 \%$ of all deaths and $77 \%$ of the European disease burden. ${ }^{1,2}$

Multi-morbid patients who require a global approach, not the current fragmented care interventions and a close connection between health and social services are progressively growing.

The chronic care model (CCM) was just introduced to face these needs. It enhances patient centricity and community social and health support, in order to keep chronic, frail, poly-pathological people at home or out of the hospital as long as possible.

Acute Complex Care Model (ACCM), object of this work, is a model of integrated management of the 
frail patients when they need acute hospital care. ${ }^{3}$ Nowadays, multiple uncoordinated specialists care for them who move from one ward to another, with dangerous loss of information and continuity. ACCM tries to overcome these problems by putting the internal medicine specialist as the only referent for that patient and the coordinator of the care team.

Internal Medicine Wards (IMWs) have a capillary diffusion in the country with slightly different missions in large, medium and small hospitals. ${ }^{4}$

\section{Internal medicine wards: activities, site and organizational model}

The essential activities and attributes of IMWs are: ${ }^{5,6}$ i) diagnosis and management of complex, polypathological, and frail patients; ii) provision of basic technological care and filter for sub-specialized and highly technological care; iii) high functional flexibility from outpatients clinic and hospice to critical or sub-intensive care; iv) specialized care in internal medicine sub-categories; v) multi-professional coordination; vi) co-management of surgical patients by different models, before and after surgery.

The IMWs activities are summarized in Table 1.

\section{Acute Complex Care Model}

Acute Complex Care Model (Figure 1) is born to be the hospital counterpart of CCM. The target population is made up of acutely ill complex and polypathological patients (AICPPs), admitted to hospital and requiring high technological resources; the mission is to improve the management of medical admis-

Table 1. Internal Medicine wards activities.

\begin{tabular}{ll}
\hline Activities & Areas \\
\hline Medical admission & High-dependency and ordinary wards \\
\hline Diagnosis-treatment of acute medical patients & High-dependency and ordinary wards \\
\hline Critical care & High-dependency wards \\
\hline Treatment of chronic poly-pathological patients (i.e., during exacerbations) & Ordinary wards \\
\hline Co-management of surgical patients & Structured consultation \\
\hline Home or residential discharge & Ordinary wards \\
\hline Hospital at home & Integrated poly-specialist medical team from hospital wards
\end{tabular}

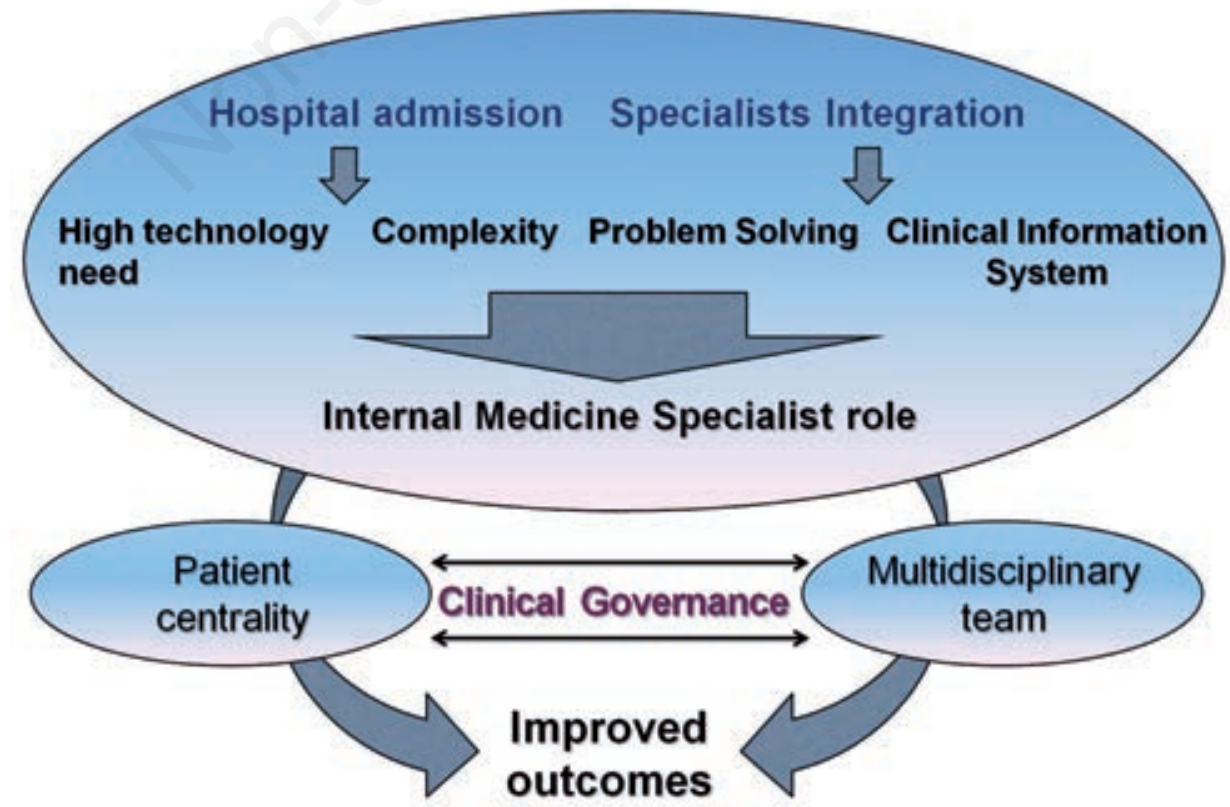

Figure 1. Acute Complex Care Model. 
sions through pre-defined intra-hospital tracks and a global, multidisciplinary, patient-centered approach. ${ }^{4}$

The ACCM strengths are: i) in-hospital care pathways, based on patients' needs and tailored for AICPPs; ii) ability to balance risks and benefits of specialist treatments for every single patient; iii) managerial competencies applied to AICPPs and multi-professional integration.

A team of internists will care for all the hospital medical patients, in all the settings from more to less intensive care, but also in surgical wards with an adequate re-distribution of resources between surgical and medical wards.

Internists trained for this job should be familiar with management of frailty and dementia, critical, complex, and comorbid patients and coordination of care. ${ }^{7}$

Ability to perform some procedures, like abdominal paracentesis, arterial line placement, advanced cardiac life support, central venous line placement, lumbar puncture, nasogastric intubation, thoracentesis ${ }^{6}$ and intercostal drain insertion, direct current cardioversion, urethral catheterization, temporary cardiac pacing, ${ }^{7}$ bed-side ultrasonography and non-invasive mechanical ventilation, are strongly recommended for ACCM internists.

\section{The distinctive features of Acute Complex Care Model}

A critical point of this model is the correct identification of the target patient: AICPPs requiring hospitalization. They are highly unstable and complex and their care pathway can involve intensive care, highdependency and/or ordinary areas, sub-acute areas and nursing home, before they return safely back home and are entrusted to primary care. The acute polypathological patient should be recognized on arrival to the hospital and before hospital admission. Alternative outpatients' pathways must be searched for, in order to enhance appropriateness of care, to avoid overcrowding of emergency and medical wards, and to reduce clinical risk. ${ }^{3}$

The distinctive features of this promising organizational approach are the following: i) proper selection of patients requiring hospitalization (risk/benefit ratio); ii) care pathways focused on patients; iii) global approach to patients; iv) problem solving and list of priorities; v) coordinated and integrated multi-disciplinary caring team; vi) appropriate use of resources (high technology, personnel, other equipment); vii) movements through different care settings depending on care needs and not on specializations; viii) reliable and comprehensive information system for clinical data sharing.

Patients should be allocated to different areas, depending on their clinical stability. ${ }^{8-11}$ Predictive scoring systems, such as modified early warning score (MEWS), Vitalpac ${ }^{\mathrm{TM}}$ early warning score (ViEWS) and national early warning score (NEWS) have been developed to measure the severity of disease and the prognosis of patients both in general wards and in Intensive Care Units (ICUs), so they can be useful for this aim. ${ }^{12-14}$ Nevertheless, whatever the score used for the stratification of patients, it can never totally replace the clinical judgment. ${ }^{15}$

A patient with multiple comorbidities but a singleorgan acute disease should be referred to an internist who, because of his/her widespread knowledge and expertise, will approach him/her globally, coordinate organ specialists if needed, and optimize poly-pharmacy. The internist in charge will write a clinical summary, the list of priorities and the composition of the multidisciplinary team, immediately after the admission., ${ }^{3,8}$

Problem solving and extensive use of information system allowing quick data networking are the base for specialists' integration. Actually, problem solving is the process by which: i) the case is analyzed on the base of clinical experience; ii) knowledge and information provided by acquired data and interaction between specialists are integrated; iii) the solution of the problem is reached as quickly and efficiently as possible.

The care must be patient-centered. The patient should not be moved from one ward to another unless it is strictly necessary (i.e., transferred from medical ward to intensive care or surgical ward). The patient should be followed up to discharge by the same medical-nursing team that will address his/her clinical but also social problems. The caring internist - the consultant - should be present every day - from admission to discharge - to ensure the continuity of care, to avoid handover errors and to warrant effective interaction with the specialists and better communication with patient and family. 3,7

ACCM attributes are displayed in Table 2.

\section{High dependency areas}

As reported in medical literature, $20 \%$ of people admitted to IMWs present deterioration of one or more organs or high risk of organ deterioration, because of which they require stricter clinical and nursing control than it is usual in ordinary wards. ${ }^{14,16}$ They are mainly patients coming from emergency room or other wards, who are not candidate for admission to ICU because of their age, and/or multiple comorbidities, but also for their less severe disease status. They are received in dedicated areas, called High Dependency Areas (HDAs), but also InterMediate Care Units (IMCU) that have shown to improve the outcome of these critical patients. In a recent multi-center European cohort study, involving 17 European countries, patients admitted to ICUs with an IMCU in the hospital, experienced sig- 
nificantly reduced mortality, when compared with patients admitted to ICUs in hospital without an IMCU. ${ }^{17}$ These areas offer a more favorable nurse/patients ratio, quicker responses to changes in clinical conditions that ensure patient safety and a more adequate allocation of resources that favors healthcare sustainability.

A clear definition of roles and responsibilities for any team component is fundamental for the efficacious and safe management of patients in these areas, because of the multidisciplinary approach. ${ }^{3}$ Table 3 shows the main diseases treated in Internal Medicine HDAs.

HDAs should be organized with flexible equipment (vital signs monitoring, non-invasive ventilation, etc.) in order to allocate resources more appropriately.

A consultant should not increase his patients' number to more than $20 \%$ (average $10-12$ patients) as it can reduce efficacy and efficiency.

In HDAs, nurses have a particularly relevant role and responsibility and should be specifically trained for monitoring systems, active surveillance, the use of defibrillators, non-invasive ventilators, infusion devices, drainages, central venous lines, arterial lines, ultrasounds, etc., as well as for the management of emergencies and urgencies according to clear, pre-defined, and shared protocols.

\section{How acute complex care model can actualize hospitals and organizational models based on intensity of treatment}

Hospital organization according to intensity of treatment is the current answer to the changed patient's characteristics such as old age, complexity, the presence of multiple active diseases. Recent articles, ${ }^{18}$ referring to IMWs, highlighted this issue and defined structural, organizational, technological, managerial, and staff-related characteristics to better determine and classify the intensity of treatment organization. To implement this organization the following actions are crucial: i) accurate identification of the medical care levels; ii) differentiated staff and technological equipment according to the level of medical care provided; iii) admission criteria for each level of medical care; iv) strict interaction between Internal Medicine Department, Emergency Department and Specialties; v) integration of different competencies.

ACCM represents an instrument to help in implementing the hospital organization according to the concept of intensity of treatment. In fact, ACCM features represent the tool box to drop in the daily prac-

Table 2. Acute complex care model attributes.

\begin{tabular}{|c|c|}
\hline Attributes & Related actions \\
\hline 1. Integrated care & $\begin{array}{l}\text { Hospital internist works as team coordinator in the care of AICPPs } \\
\text { requiring hospital care for acute diseases }\end{array}$ \\
\hline 2. Creation of a multi-professional team & $\begin{array}{l}\text { Hospital internist promotes and manages the multi-professional } \\
\text { team, including organ specialists, nurses and other professionals to } \\
\text { cover patient health needs in and out of the hospital }\end{array}$ \\
\hline 3. Medical wards with progressive care & $\begin{array}{l}\text { Setting up of areas according to care intensity: } \\
\text { High dependency area for unstable patients } \\
\text { Ordinary area for acute stable patients } \\
\text { Post-acute area for stable patients who cannot be discharged yet }\end{array}$ \\
\hline 4. Constructive alliance with caregivers & $\begin{array}{l}\text { Medical-nursing team defines patients' in-hospital care pathway, } \\
\text { needs for sub-specialized care and other care needs, creating a con- } \\
\text { structive alliance with caregivers to ensure an adequate follow-up } \\
\text { after the acute phase }\end{array}$ \\
\hline 5. Best scientific evidence and clinical competence & $\begin{array}{l}\text { Treatments should constantly reflect the best scientific evidence and } \\
\text { all the health professionals should ensure high competence levels, } \\
\text { constant updating and continuous improvement }\end{array}$ \\
\hline 6. Extensive utilization of informative system for clinical data sharing & $\begin{array}{l}\text { The model requires a complete integration of information during the } \\
\text { hospital admission phase. Extensive utilization of an informative sys- } \\
\text { tem to trace any intervention and to share patient's health information } \\
\text { It also represents the first step in hospital-community integration. } \\
\text { Information system will provide: } \\
\text { Alerts for sentinel events to help caring team to follow guidelines } \\
\text { Constant clinical data outline } \\
\text { Disease database } \\
\text { Database management } \\
\text { Care pathway tracking }\end{array}$ \\
\hline
\end{tabular}

AICPPs, acutely ill complex and poly-pathological patients. 
tice the intensity of care applied at hospital level and in medical area. It is clear that, the implementation of a new organizational model must be careful and consider the possible enlargement errors that can be made. A preliminary context analysis is necessary to assess prerequisites, excluding the elements opposed to the success of the proposed model such as overcrowding or inadequate ratio between number of patients and health staff, with a consequential increased risk of adverse events. Before implementing new models, it is mandatory to perform an epidemiological assessment in order to better understand the patients' needs and the level of complexity/criticality/instability. ${ }^{19}$

Table 3. Main diseases treated in Internal Medicine high dependency areas.

Severe type I-II respiratory failure, not requiring invasive ventilation

Severe acute pancreatitis

Pulmonary edema

Heart failure and co-morbidities

Diabetic ketoacidosis

Hyperglycemic, hyperosmolar nonketotic coma

Life-threatening ions alterations

Hypo- and hyperkinetic arrhythmia

Acute coronary syndromes

Stroke

Severe sepsis and septic shock in patients with multiple comorbidities

Gastrointestinal bleeding with hemodynamic instability

Massive pulmonary embolism

Pre- and post-surgical medical complications

Any condition with severe failure of organs or systems

\section{Organizational standards}

Staff and technology requirements will have to be suitably adapted to the needs of clinical care, because of the wide variability of Internal Medicine wards in the various hospitals. Variability depends on: i) type of patients, mean age, case mix, percentage of bedridden and/or dependent patients, need for non-invasive ventilation, infusion pumps or complex diagnostic procedures (endoscopy, angiography, etc.); ii) turn-over rate, mean length of stay and occupancy rate; iii) day-time, night-time, week and holidays doctor's coverage, the need to perform some shifts in the emergency department; iv) presence of students or specialists in training.

An update of Internal Medicine health personnel standards is advisable, given the characteristics of hospitalized patients and the changing organizational modalities according to the intensity of care criteria.

Table 4 shows the General Requirements and Technological equipment suggested for HDAs, according to the recent Emilia Romagna Region's document on minimum requirements for accreditation of IMWs. ${ }^{20}$ In the following paragraphs, we propose medical staff requirements for the different internal medicine settings.

\section{High dependency areas}

It is important the presence of one doctor (tutor) during daytime. At night and on public holidays, the medical service can be shared with the rest of the internal medicine ward or the medical department, on availability.

The health personnel to be allocated to these areas have been defined in 2012 by Emilia Romagna Region indicating at least 2 nurses per shift each 30 beds $24 \mathrm{~h}$ a day, 7 days out of 7 and an additional unit per shift (nurses or Social Health Operator - OSS) every 15 ad-

Table 4. General requirements and technological equipment in high dependency areas.

\section{General requirements}

Monitored beds should be at least $10 \%$ of total beds

Inside Internal medicine area

Equipped with a monitoring unit or visual control system

Adequate space around the bed to ensure nursing maneuvers

Patient privacy guaranteed

\section{Special equipment}

$100 \%$ poly-parametric monitored beds, ECG monitor, non-invasive blood pressure, $\mathrm{O}_{2}$ saturation, parameters trend, audible and visual alarms

Beds connected with centralized system and with the possibility of Trendelenburg and anti-Trendelenburg position

For each bed, early warning system, $\mathrm{O}_{2}$ availability: 1 for vacuum and 1 for compressed air

Defibrillator and pacemaker monitors

Electrocardiograph

Availability of syringes and volumetric infusion pumps (equal to the number of beds)

Blood gas analyzer available $24 \mathrm{~h}$ a day (continuously) also shared with other hospital services

Intubation set/cardio pulmonary resuscitation

At least half of monitored bed equipped with non-invasive ventilators

ECG, electrocardiogram. Modified from Agenzia Sanitaria Regionale Emilia Romagna, 2012.20 
ditional beds. A doctor on duty must be present $24 \mathrm{~h}$ a day, 7 days out of 7, possibly shared with other Medical Units. ${ }^{20}$ Taking in account the standards adopted by Emilia Romagna and integrating them with consensus meetings held in Liguria Region (Gruppo di lavoro Controllo di Gestione Sanitaria, Modello sperimentale di misurazione dell'attività del personale medico delle Aziende del SSR - 9 mesi 2014; unpublished work), the proposed standards are: 3 doctors (2 doctors in the morning and 1 in the afternoon) during the 12 daytime hours, every 10-12 beds. For nursing the requirement is of at least 1 nurse every 6 patients.

About support staff, 1 OSS every 10-12 patients.

We believe that $300-360 \mathrm{~min} /$ patient/day is adequate for the care needs of this area. According to these assumptions, one can imagine that in a ward of 10-12 beds, there are 2 nurses and 1 OSS per shift, with an additional nurse case manager in the morning.

\section{Areas for acute stable patients (ordinary wards)}

In these areas, care needs are less complex and intensive, so we suggest: i) 4 doctors for a ward of 20 beds, equaling internal Medicine with other medical specialties, according to the recent AGENAS (Agenzia Nazionale per $i$ Servizi Sanitari Regionali) standards ${ }^{21}$ ii) for nurses and OSS, we assumed that 195 minutes/patient/day of care, stated by Veneto Region ${ }^{22}$ are insufficient and 255 minutes/patient/day are appropriate. They correspond to: 2 nurses and 2 OSS in the morning and in the afternoon, and 2 nurses and 1 OSS at night, in a ward of 20 beds.

\section{How the model could work in the Italian Internal Medicine Wards}

According to the recent study on 2013 hospital discharge records, ${ }^{23} 55 \%$ of Italian hospital admissions are emergency admissions. Hospitalization rates in emergency are systematically higher than those in election and the greatest differences are in the regions with inefficiently organized regional network.

The role of the hospital Internal Medicine appears crucial in providing beds to the Emergency Room, in fact it receives $27 \%$ of all urgent admissions. Determinants significantly affecting the emergency admissions are old age and co-morbidities of the patients that also increase the length of hospital stay (LOS). In this scenario, ACCM, improving the management of medical admissions through pre-defined intra-hospital tracks and a global, multidisciplinary, patient-centered approach and having as target AICPPs admitted to hospital and requiring high technological resources and continuous monitoring, could be a valuable proposal for the organization of the future hospital.

Moreover, in a perspective of hospital based on progressive care, the stratification of the patients using validated scores, the definition of a HDA (needed by $20 \%$ of admitted patients in unstable conditions), the amelioration of the clinical competence for doctors and nurses working in Internal Medicine wards and the integration of different specialists could reasonably improve the clinical management of the complex patients reducing risks and shortening LOS.

\section{Discussion}

It is well known that an urgent health systems reorganization is required. In this scenario, we, internal medicine specialists, feel a sort of disconnection between our hospital work and the role attributed to hospital IM by policy makers and health managers.

Internist's role, as it is demonstrated by official documents where the discipline is always named General Medicine, is not clear to decision-makers who often have the idea that patients admitted to IMWs can also be managed out of the hospital. Therefore, government promotes out of hospital care pathways for chronic and poly-pathological patients in order to reduce hospital admission and leave hospital to acute patients requiring organ specialists' care. Anyway, every day hospital practice is completely different with a lot of acute comorbid patients who cannot be managed by organ specialists, alone, but ask for a holistic approach by a specialist of complexity, such as the internist. The IMWs overcrowding forced patients turn-over and the outliers confirm this unsustainable situation.

Among the objectives of a scientific society there is also an active, practice-based support to health policy in order to drive organizational changes that reduce waste but do not penalize patients and professionals. As a constant increase of acute poly-pathological complex patients is reported everywhere, it is vital to enhance hospital's Internal Medicine, enlarging and strengthening the medical areas where highly trained internists, assisted by organ specialists, can offer appropriate care for these patients.

Our work describes a new organizational model just for acute poly-pathological and complex patients: the ACCM, addressing the management of these patients, who can easily lead to exaggerated resource consumption, without a proper multi-professional coordination and risk/benefit assessment that only an internist is able to realize.

The name of our model - ACCM - just recalls the $\mathrm{CCM}$, designed for the de-hospitalization of chronic patients by preventing acute exacerbations. In fact, ACCM represents the hospital counterpart of the CCM.

Updating of medical and nursing staff of internal medicine wards - dated back to 1988 - is essential for the efficacious and efficient management of the polypathological patients admitted to hospital for acute 
diseases. ${ }^{24}$ Hundreds and hundreds of outliers every year require a mandatory revision of hospital beds distribution between Internal Medicine and other specialties. Internal Medicine cannot be considered as a basic specialty any more. It cares for acute, sometimes unstable, multi-morbid and so complex patients requiring more time and more intense care than patients with a mono-organ disease.

\section{Conclusions}

The epidemiological transition leading to a progressive increase in chronically unstable patients requiring frequent hospital treatments inevitably enhances the role of hospital internal medicine specialist in the coordination and delivery of care. The ACCM represents a practical response to this epochal change of roles. Medical and nursing staff standards and competencies have to be reviewed to ensure adequate and trained care for these patients.

\section{References}

1. World Health Organization (WHO). Health systems response to NCDs. Available from: http://www.euro.who. int/en/health-topics/Health-systems/health-systems-response-to-ncds Accessed: April 24, 2015.

2. World Health Organization (WHO). Better non communicable disease outcomes: challenges and opportunities for health systems. Assessment Guide. Geneva: WHO Regional Office for Europe; 2014. Available from: http://www.euro.who.int/en/health-topics/Health-systems/health-systems-response-to-ncds/publications/ 2014/better-noncommunicable-disease-outcomes-challenges-and-opportunities-for-health-systems.-countryassessment-guide-2014

3. Pietrantonio F, Orlandini F, Moriconi L, La Regina M. Acute Complex Care Model: an organizational approach for the medical care of hospitalized acute complex patients. Eur J Med 2015;26:759-65.

4. Porcel JM, Casademont J, Conthe P, et al. Core competencies in internal medicine. Eur J Intern Med 2012; 23:338-41.

5. Scotti E, Pietrantonio F. The hospital Internal Medicine specialist today: a literature review and strength, weaknesses, opportunity, threats (SWOT) analysis to develop a working proposal. Ital J Med 2013;7:278-86.

6. La Regina M, Orlandini F, on behalf of the Ligurian FADOI group. Census of Ligurian Internal Medicine Wards of nonteaching hospitals. Ital J Med 2014;8:246-52.

7. Future Hospital Commission (FHC). Future Hospital: caring for medical patients. London: Royal College of Physician; September 2013. Available from: https:// www.rcplondon.ac.uk/projects/outputs/future-hospitalcommission

8. Nardi R, Mathieu G, Berti F, et al. The working group FADOI-SDA BOCCONI. Evaluation models and items of clinical competence for the hospital physicians in internal medicine. Ital J Med 2011;55:S3-S13.
9. Flanders SA, Wachter RM. Hospitalists: the new model of inpatient medical care in the United States. Eur J Intern Med 2003;14:65-70.

10. Kellett J. Acute hospital medicine - a new sub-speciality or internal medicine re-born? Eur J Intern Med 2011; 22:334-8.

11. Nardi R, Berti F, Fabbri LM, et al. Towards a sustainable and wise healthcare approach: potential contributions from hospital Internal Medicine Departments to reduce inappropriate medical spending. Ital J Med 2013;7:65-81.

12. Passalia C, Mazzacurati G, Marinaro A, et al. MEWS, RAY score and resources consumptions. Ital J Med 2014;8:97-8.

13. Prytherch D, Smith GB, Schmidt PE, Featherstone PI. ViEWS-towards a national Early Warning Score for detecting adult inpatient deterioration. Resuscitation 2010; 81:932-7.

14. Kellet J, Kim A. Validation of an abbreviated Vital-

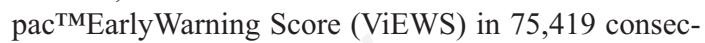
utive admissions to a Canadian regional hospital. Resuscitation 2012;83:297-302.

15. Chesi G, Nardi R. Severity Stratification of patients hospitalized on the Internal Medicine ward: work in progress. Ital J Med 2013,7:231-3.

16. Bartolomei C, Cei M. The allocation of patients in an intensity- of-care based Internal Medicine ward: the ADOIT Tri-Co (Triage in the Corridor) study. Ital J Med 2007;2:31-9.

17. Capuzzo M, Moreno RP, Le Gall JR. Outcome prediction in critical care: the Simplified Acute Physiology Score models. Curr Opin Crit Care 2008;14:485-90.

18. Chesi G, Boni F. Ospedali e modelli organizzativi per intensità di cure: il punto di vista dell'internista. Ital J Med 2012;6:63-71.

19. Nardi R, Arienti V, Nozzoli C, Mazzone A. Organizzazione dell'ospedale per intensità di cure: gli errori da evitare. Ital J Med 2012;6:1-13.

20. Agenzia Sanitaria Regionale Emilia Romagna. Requisiti specifici per l'accreditamento delle strutture di Medicina Interna. Delibera ${ }^{\circ} 419$ del 10/04/2012. Available from: http://assr.regione.emilia-romagna.it/it/servizi/normativa/autorizzazione-e-accreditamento/normativa-regionale/requisiti-specifici-accreditamento/req-s-medicinainterna

21. Agenzia Nazionale per i Servizi Sanitari Regionali (AGENAS). Monitor - Elementi di analisi e osservazione del sistema salute. 2011;X(27). Available from: http://www. quotidianosanita.it/allegati/allegato2497031.pdf

22. Regione Veneto - Deliberazione della Giunta Regionale n. 610 del 29 aprile 2014 Definizione dei valori minimi di riferimento per il personale di assistenza del comparto dedicato alle aree di degenza ospedaliera. Articolo 8, comma 1, della L.R. 29 giugno 2012, n. 23. Deliberazione n. 179/ CR del 30 dicembre 2013. Available from: http://bur.regione.veneto.it/BurvServices/pubblica/DettaglioDgr.aspx?id=273829

23. Pietrantonio F, Piasini L, Spandonaro F. Internal Medicine and emergency admissions: from a national Hospital Discharge Records (SDO) study to a regional analysis. Ital J Med 2016;10:157-67.

24. La Regina M, Orlandini F. Internal Medicine wards and the chronic diseases epidemic: it is time to change the standards. Intern Emerg Med 2014;9:483-5. 